\title{
Preliminary evaluation of dynamic glucose enhanced MRI of the human placenta during glucose tolerance test
}

\author{
Jie Luo ${ }^{1,2}$, Esra Abaci Turk ${ }^{2}$, Borjan Gagoski ${ }^{2}$, Natalie Copeland ${ }^{2}$, Iris Y. Zhou ${ }^{3}$, Vanessa Young ${ }^{2}$, \\ Carolina Bibbo $^{4}$, Julian N. Robinson ${ }^{4}$, Chloe Zera ${ }^{4}$, William H. Barth Jr ${ }^{5}$, Drucilla J. Roberts ${ }^{6}$, \\ Phillip Zhe Sun ${ }^{3,7}$, P. Ellen Grant ${ }^{2}$
}

${ }^{1}$ School of Biomedical Engineering, Shanghai Jiao Tong University, Shanghai 200240, China; ${ }^{2}$ Fetal-Neonatal Neuroimaging and Developmental Science Center, Boston Children's Hospital, Boston, MA, USA; ${ }^{3}$ Athinoula A. Martinos Center for Biomedical Imaging, Department of Radiology, Massachusetts General Hospital, Charlestown, MA, USA; ${ }^{4}$ Department of Obstetrics and Gynecology, Brigham and Women's Hospital, Boston, MA, USA; ${ }^{5}$ Department of Obstetrics and Gynecology, ${ }^{6}$ Department of Pathology, Massachusetts General Hospital, Boston, MA, USA; ${ }^{7}$ Department of Radiology and Imaging Sciences, Emory University School of Medicine, Atlanta, GA, USA

Correspondence to: Phillip Zhe Sun, PhD. Department of Radiology and Imaging Sciences, Emory University School of Medicine, Atlanta, GA 30329, USA. Email: pzhesun@emory.edu; P. Ellen Grant, MD, MSc. Fetal-Neonatal Neuroimaging and Developmental Science Center, Boston Children's Hospital, Boston, MA 02115, USA. Email: Ellen.Grant@childrens.harvard.edu.

Background: To investigate dynamic glucose enhanced (DGE) chemical exchange saturation transfer (CEST) MRI as a means to non-invasively image glucose transport in the human placenta.

Methods: Continuous wave (CW) CEST MRI was performed at 3.0 Tesla. The glucose contrast enhancement (GCE) was calculated based on the magnetization transfer asymmetry (MTRasym), and the DGE was calculated with the positive side of Z-spectra in reference to the first time point. The glucose CEST (GlucoCEST) was optimized using a glucose solution phantom. Glucose solution perfused ex vivo placenta tissue was used to demonstrate GlucoCEST MRI effect. The vascular density of ex vivo placental tissue was evaluated with yellow dye after MRI scans. Finally, we preliminarily demonstrated GlucoCEST MRI in five pregnant subjects who received a glucose tolerance test. For human studies, the dynamic R2* change was captured with $\mathrm{T}^{*}$-weighted echo planar imaging (EPI).

Results: The GCE effect peaks at a saturation B1 field of about $2 \mu \mathrm{T}$, and the GlucoCEST effect increases linearly with the glucose concentration between 4-20 mM. In ex vivo tissue, the GlucoCEST MRI was sensitive to the glucose perfusate and the placenta vascular density. Although the in vivo GCE baseline was sensitive to field inhomogeneity and motion artifacts, the temporal evolution of the GlucoCEST effect showed a consistent and positive response after oral glucose tolerance drink.

Conclusions: Despite the challenges of placental motion and field inhomogeneity, our study demonstrated the feasibility of DGE placenta MRI at 3.0 Tesla.

Keywords: Dynamic glucose enhancement; glucose chemical exchange saturation transfer (GlucoCEST); MRI; placental function

Submitted Jun 13, 2019. Accepted for publication Sep 09, 2019.

doi: 10.21037/qims.2019.09.16

View this article at: http://dx.doi.org/10.21037/qims.2019.09.16

\section{Introduction}

Placental glucose transfer is essential to sustain fetal development (1). Insufficient glucose transfer may result in intrauterine growth restriction (IUGR) while too much glucose is associated with fetal macrosomia. Such conditions can further lead to complications at birth and an increased burden of chronic diseases in adulthood (1). The fetal glucose level varies with the gestational age and the 
maternal glucose level. In addition, diabetes and gestational diabetes have been associated with elevated maternal glucose levels and risk of birth complications. Whereas the glucose tolerance test screens for gestational diabetes (2), it does not provide information about placental glucose transfer. Routine ultrasound exams are not are not able to characterize maternal-fetal glucose relationships. Although PET can map tracer distribution across the placenta and in fetal organs in experimental animals, it is not feasible in humans due to the ionizing radiation (3). Hence, it is urgent to develop a safe non-invasive means to assess placental glucose transport.

Advanced MRI techniques are being investigated for placental imaging. For example, placental perfusion can be measured by dynamic contrast-enhanced (DCE) MRI $(4,5)$, arterial spin labeling (ASL) (6) and intravoxelincoherent motion (IVIM) MRI (7-9). In addition, placental oxygenation can be assessed using $\mathrm{T} 2$ and $\mathrm{T} 2$ * mapping $(10,11)$ and BOLD MRI (12-14). In addition, MR spectroscopy (MRS) has be used to study the placenta (15-17) but its spatial resolution is limited. Recently, chemical exchange saturation transfer (CEST) MRI has been shown to be glucose sensitive via the exchange between bulk water and the glucose hydroxyl protons, dubbed GlucoCEST MRI $(18,19)$. Dynamic glucose enhanced (DGE) MRI provides time-resolved glucose measurement in brain tumors at high field $(20,21)$, which has been extended for head and neck cancer imaging at 3.0 Tesla (22). Recently, Wu et al. demonstrated DGE MRI at 11.7 Tesla in a mouse model of intrauterine inflammation (IUI), which is known to result in acute placental injury (23). It is necessary to note that most of these DGE experiments were performed with intravenous (IV) glucose administration.

Although it is advantageous to perform CEST MRI at high field (24), it is important to develop GlucoCEST MRI at 3.0 Tesla, particularly for the pregnant population. For patient acceptance, getting MRI scan after an oral glucose drink is far preferable to an IV load. Oral glucose tolerance test is commonly used during pregnancy to screen for gestational diabetes. To address these two issues, our study here optimized continuous wave (CW) GlucoCEST echo planar imaging (EPI) at 3.0 Tesla (25). We tested GlucoCEST in ex vivo placentas and characterized the relationship between GlucoCEST and placental vascular density. We also demonstrated GlucoCEST MRI following the glucose tolerance test and established the feasibility of placental DGE as an ancillary exam.

\section{Methods}

The study has been approved by the Institutional Review Board (IRB) at the Boston Children's Hospital and Massachusetts General Hospital.

\section{Glucose phantom study}

D-glucose was dissolved in PBS at concentrations of 4, 8, 12 and $20 \mathrm{mM}$ with $\mathrm{pH}$ titrated to 7.4. The solution was transferred into separate $50 \mathrm{~mL}$ Falcon tubes, and positioned in a cylindrical phantom holder.

\section{Ex vivo placental tissue study}

Fresh placentas were obtained from uncomplicated term deliveries within $6 \mathrm{~h}$. Cannulation was performed on the surface fetal chorionic arteries of the placenta specimen. The lobules were flushed with normal saline and $5,000 \mathrm{IU} / \mathrm{L}$ heparin $(\mathrm{NSH})$ until the venous return was clear. Subsequently, NSH supplemented with D-glucose $(5,10,20 \mathrm{mM})$ and $1 \mathrm{~mL} / \mathrm{L}$ yellow India ink (Cancer Diagnostics, Inc.) were perfused in separate placenta lobules of a single placenta. After the perfusion, the area around the perfused lobule was clamped, sutured, and fit into a cylindrical plastic container of $10 \mathrm{~cm}$ diameter. Four placenta sections were prepared and stacked in the container, which was then filled with agar gel (1\%) (Figure 1). The sample was maintained at room temperature before imaging. Hematoxylin and Eosin histology was obtained after MR imaging and examined by an experienced placenta pathologist.

\section{In vivo study}

The GlucoCEST scans were performed in pregnant mothers (Table 1). After a glucose drink $\left(\right.$ Trutol $\left.^{\mathrm{TM}}\right)$ in a sitting position with a dose of $50 \mathrm{gm}$, equivalent to glucose tolerance test, subjects re-entered the scanner while keeping the same maternal position for the dynamic GlucoCEST scan. $\mathrm{T}_{2}{ }^{*}$-weighted scans were performed simultaneously.

\section{Data acquisition}

All studies were performed on a 3.0 Tesla Skyra (Siemens Healthcare, Erlangen, Germany). While phantom and ex vivo tissue was scanned using a 32-channel array head coil, in vivo subjects were scanned using a combined 

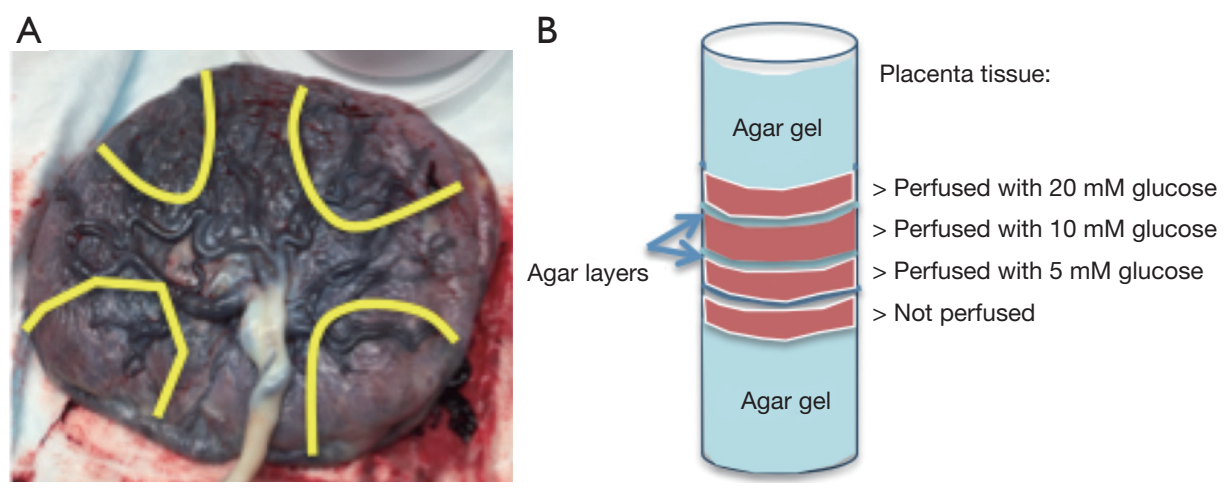

Figure 1 Sample configuration of the placental tissue study. (A) The fetal surface of the placenta, yellow lines indicate where the placenta was sectioned after perfusion. Each segment and its glucose concentration were labeled; (B) illustration of perfused placental tissue positioned in a cylindrical container.

Table 1 Subject demographics

\begin{tabular}{llll}
\hline Subject & Gestational age & Position & Fast $(\mathrm{h})$ \\
\hline 1 & 33 weeks & Left lateral & 2 \\
2 & 35 weeks & Left lateral & 2 \\
3 & 30 weeks $+5 \mathrm{~d}$ & Left lateral & 2 \\
4 & 34 weeks $+6 \mathrm{~d}$ & Right lateral & 2 \\
5 & 35 weeks & Left lateral & 2 \\
\hline
\end{tabular}

18-channel body and 12-channel spine receive arrays. CEST imaging was performed with single-shot gradient echo EPI (26) with a CW RF irradiation of 1 second. For the phantom study, we repeated CEST MRI measurements under $\mathrm{B}_{1}$ fields of $1,1.5,2$ and $3 \mu \mathrm{T}$, and flip angle (FA) of 20, 45, 70 and 90 degrees. Z-spectra were obtained with $\mathrm{RF}$ saturation frequency from -5 to $5 \mathrm{ppm}$ with intervals of $0.25 \mathrm{ppm}$. Imaging matrix $48 \times 48$; in-plane resolution $3 \times 3 \mathrm{~mm}^{2}$, slice thickness $10 \mathrm{~mm}$; TR/TE $=4 \mathrm{~s} / 17 \mathrm{~ms}$, $\mathrm{FA}=90^{\circ}, \mathrm{BW}=2.3 \mathrm{kHz} / \mathrm{px}$, averages $=2$. WASSR scan was performed with the same FOV and resolution as CEST scan at $\mathrm{B} 1=0.5 \mu \mathrm{T}$. For the ex vivo experiment, double spin echo MRI was performed for $T_{2}$ imaging ( $T R=1 \mathrm{~s}$, $\mathrm{FA}=90$ degree, TE1/TE2 $=8.5 / 50 \mathrm{~ms}$ ). In addition, $\mathrm{T}_{1}$ was calculated based on images acquired with a double FA gradient echo sequence $(\mathrm{TR} / \mathrm{TE}=15 / 1.7 \mathrm{~ms}$, FA1/FA2 $=5 / 26$ degrees). Phantom and ex vivo experiments were performed at room temperature $\left(25^{\circ} \mathrm{C}\right)$.

For in vivo study, all scans were performed freebreathing. The baseline (before glucose drink) CEST image was collected with a CW CEST MRI (RF saturation time $\left.=1 \mathrm{~s}, \mathrm{~B}_{1}=1.5 \mu \mathrm{T}\right)$. Upon mother re-entering the scanner, the dynamic CEST scan was performed with saturation frequency sweep densely between 0.75 and $4 \mathrm{ppm}$ with intervals of $0.25 \mathrm{ppm}$, and sparsely between -4 to 0 with intervals of $2.0 \mathrm{ppm}$. Each dynamic scan has a total of 17 frequency offsets plus two reference scans without on resonance saturation, which adds up to $1 \mathrm{~min} 16 \mathrm{~s}$ for each dynamic scan. At the end of the scan, a full CEST $\mathrm{z}$-spectrum was acquired. A field map was collected with a double gradient echo MRI.

\section{Data processing}

$\mathrm{B}_{0}$ map was obtained by Lorentzian fitting of z-spectrum, followed by Water saturation shift referencing (WASSR) correction of z-spectrum (27). ROIs of placental perfusion regions (and phantom tubes) were manually outlined and magnetization transfer asymmetry $\left(\mathrm{MTR}_{\text {asym }}\right)$ [defined as $M_{\text {sat }}(-)-M_{\text {sat }}(+) / M c t r l$, where Mctrl is the control scan without saturation, $M_{\text {sat }}(-)$ and $M_{\text {sat }}(+)$ are saturated scans with $\mathrm{RF}$ irradiation applied on negative and positive ppms, respectively] was plotted for each ROI. The GlucoCEST enhancement (GCE) is defined as the change in the integral of $\mathrm{MTR}_{\text {asym }}$ between 0.75 and $4 \mathrm{ppm}$. The DGE is defined as the change in integral of $Z$ spectra between 0.75 and $4 \mathrm{ppm}$ with reference to the $Z$ spectrum of the first time point. The reference scans of each dynamic CEST acquisition, which are T2*-weighted EPI images, were used to obtain the dynamic change of $\mathrm{R} 2^{*}$, as $\Delta \mathrm{R} 2^{*}=\log \left(\mathrm{S}_{0} / \mathrm{S}_{0}\right.$ baseline $) / \mathrm{TE}$. Therefore $\mathrm{R} 2 *$ changes were recorded concurrently as GCE changes. 

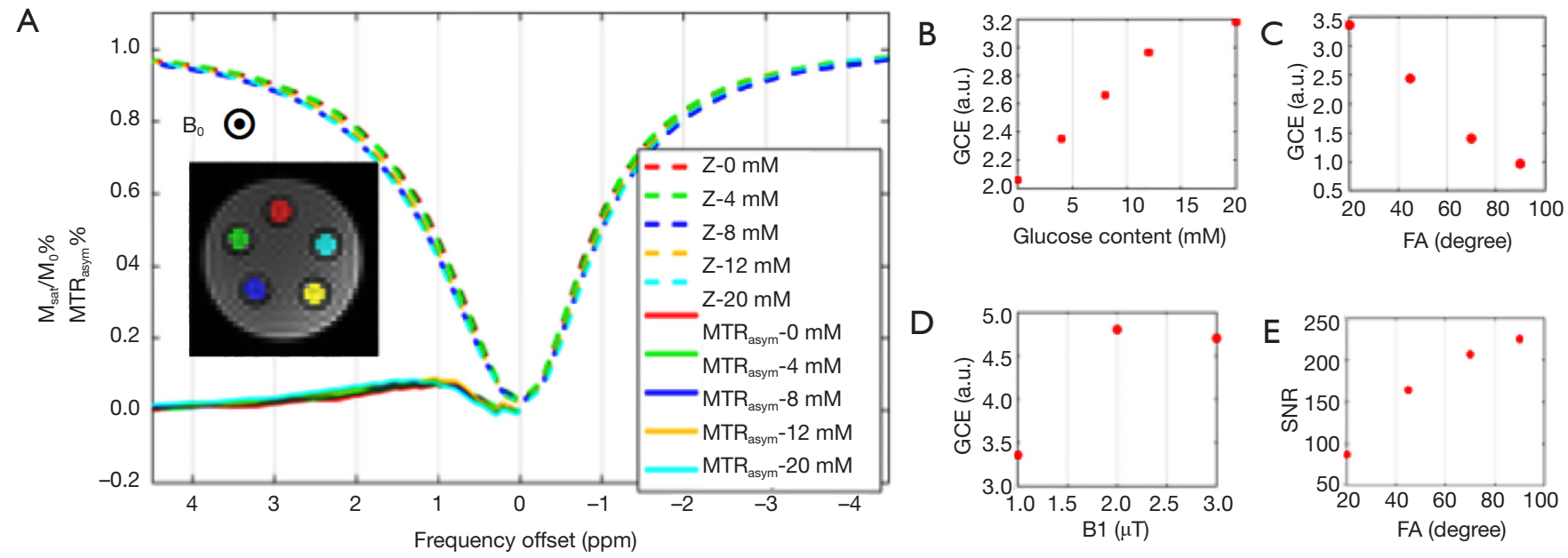

Figure 2 A phantom study of the association between GlucoCEST effect and sequence parameters in glucose solutions. (A) z-spectra at various glucose concentrations; (B,C,D) the dependence of GlucoCEST effect on glucose concentration, FA and B1 respectively; (E) SNR of the imaging data is also dependent on the flip angle. FA, flip angle; GlucoCEST, glucose chemical exchange saturation transfer; SNR, signal-to-noise ratio.

\section{Results}

Figure 2 shows the phantom GlucoCEST MRI. As shown in Figure 2B, the GCE effect increases linearly with glucose concentration. Moreover, the GCE peaked when $B_{1}=2 \mathrm{uT}$, consistent with previous simulation results (25). The FA affects the magnitude of GlucoCEST MRI effect and its signal-to-noise ratio (SNR) in opposite ways, and this finding was used as a reference in optimization placental tissue GlucoCEST MRI.

In the ex vivo placenta, the sub-regions of perfused tissue were identified based on the $\mathrm{T} 2{ }^{*}$ weighted image (Figure $3 A$ ). CEST effect in the non-perfused tissue may be due to blood sugar in the fetal vasculature $(10 \% \mathrm{v} / \mathrm{v})(28)$. The normal serum glucose level in the placenta is reported to be around $4 \mathrm{mM}$ in vivo (29). Glucoseperfused placental tissue showed higher GlucoCEST effect than the non-perfused control placental tissue (Figure $3 B$ ). This observation is also consistent with previous work that compared GCE effect in "glucose perfused", "non-perfused", and "saline perfused" placenta tissue (30). However, the GlucoCEST MRI effect did not scale linearly with the glucose perfusion concentration (Figure 3C). Notably, the section of placenta tissue perfused with $10 \mathrm{mM}$ glucose was found to have chronic villitis (Figure 3D), which may explain its lower GCE. Nevertheless, the tissue perfused by $20 \mathrm{mM}$ glucose showed only slightly higher GCE than that of $5 \mathrm{mM}$ glucose may be due to normal variations in packing density of villi and the number of vessels/capillaries per villi, which could be a major confounding factor when inferring glucose content from the GCE intensity.

The in vivo experiments were performed in five volunteers whose gestational age ranged from 30 to 35 weeks. The duration of the dynamic scans varied for each subject, from 10 to $18 \mathrm{~min}$, due to the different time needed to re-position the imaging field of view to match the baseline scan, while the maximum stay in the scanner for each subject was fixed. Nonetheless, the dynamic scans were kept within $30 \mathrm{~min}$ of the glucose drink. Example of the z-spectra of one subject is shown in Figure $4 A$. The baseline scans that were taken before glucose drink resulted in sizable variations of the GCE (-0.8 to 1.8 ) among individuals. Given the subject position changes between the baseline and the dynamic scans, the DGE of glucose was reported in reference to the first dynamic scan (Figure $4 B$ ). And the tendency of DGE increase during the first $30 \mathrm{~min}$ within glucose drink is consistent for all five subjects. Further, the $\Delta \mathrm{R} 2$ * was also assessed by dynamic $\mathrm{T} 2$ * weighted images in reference to the first time point after the glucose drink (Figure $4 C$ ). The time series of $\Delta \mathrm{R} 2 *$ and the time series DGE exhibit similarities in most subjects, however, they do not have significant correlations. 

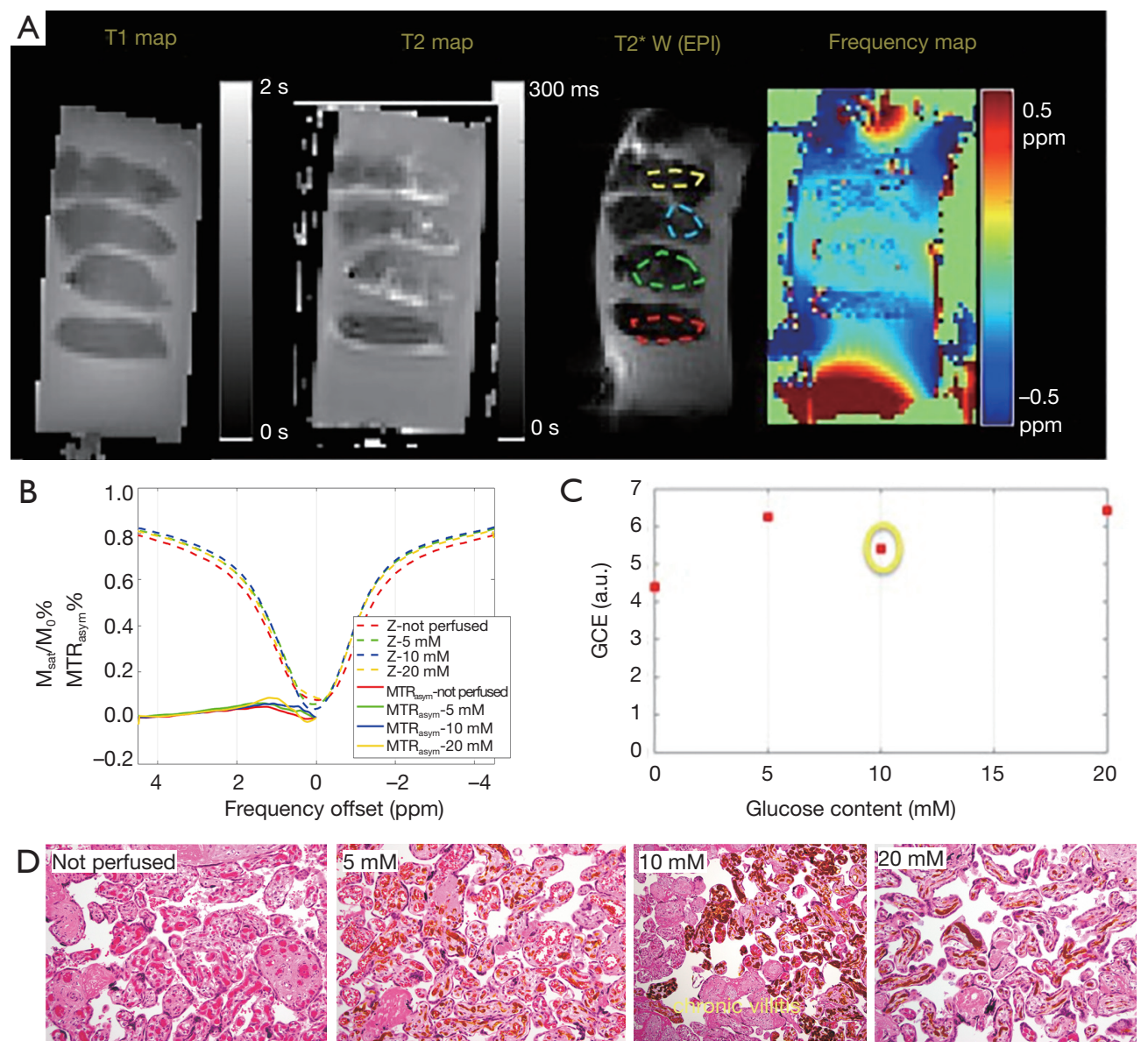

Figure 3 The GlucoCEST MRI from perfused ex vivo placenta. (A) Sagittal view of the specimen [T1map, T2map, T2* EPI (as the reference scan of CEST-EPI sequence), and B0 map]. The ROIs outlined in the perfused region; (B) z-spectra of the placenta tissue corresponding to each ROI in Figure 3C; (C) the GCE effect plotted vs. glucose concentration of the perfusate; (D) histological slides of each perfused placenta tissue, labeled with different glucose concentration, and one piece of non-perfused tissue on the left. The yellow dye was used to validate the effectiveness of perfusion, and is evident in the fetal chorionic vessels admixed with blood. The formalin fixed paraffin embedded placental samples were stained with hematoxylin and eosin, and photographed using a Olympus BX41 microscope with 100x magnification.EPI, echo planar imaging; MTR, magnetization transfer ratio; GCE, the glucose contrast enhancement; GlucoCEST, glucose chemical exchange saturation transfer.

\section{Discussion}

Our study investigated GlucoCEST MRI at 3.0 Tesla and preliminarily tested DGE MRI in human placenta following oral glucose tolerance test. The DGE signal increase was observed for each individual within $30 \mathrm{~min}$ after the glucose drink. We have demonstrated the feasibility of GlucoCEST as a novel imaging test for characterizing placental glucose transport.

The ex vivo study demonstrated that GCE is promising for detection of placental pathologies. Glucose perfused tissue exhibited higher GCE than the non-perfused tissue. Although we did not show a simple linear relationship between GCE and glucose content, the results could be partially explained by the histological findings of variable tissue perfusion possibly affected by localized placental pathology. The vessel perfusion and vascular density in the placenta tissue directly affect the volume fraction of fetal blood in a single imaging voxel and thus affect the magnitude of GCE effect. Because the maternal/fetal glucose gradient is only $1.22 \mathrm{mM}$ (fetal $3.48-3.87$ and 
A

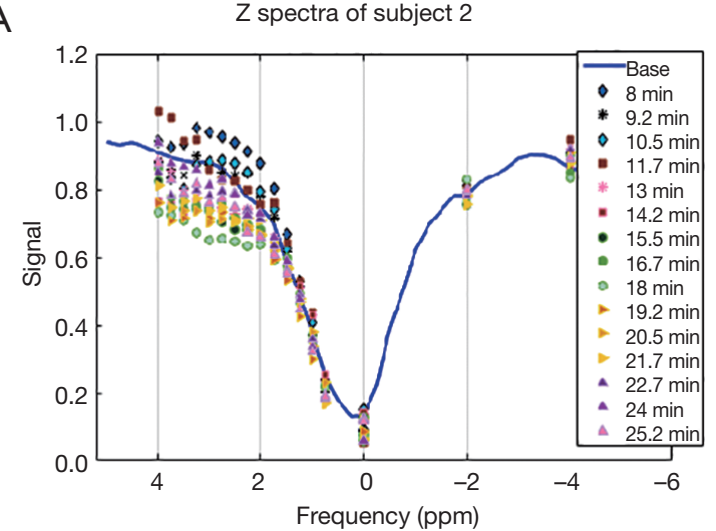

C

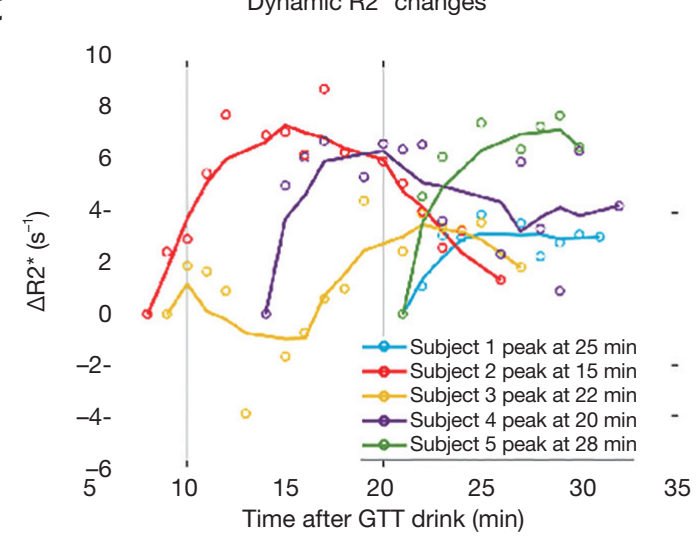

B

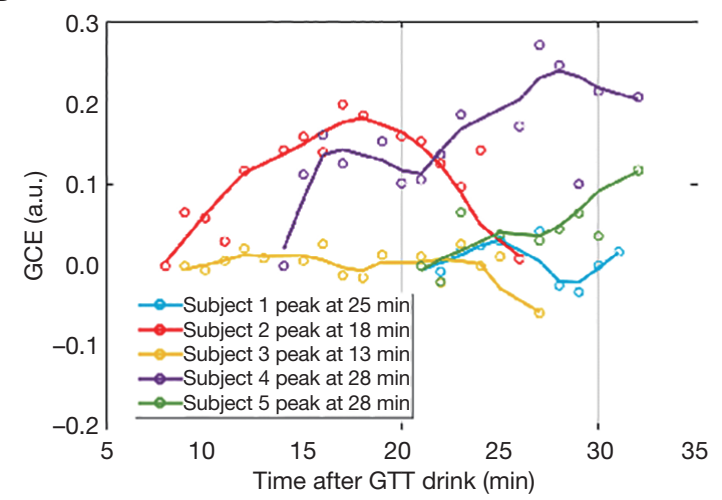

Figure 4 In vivo DGE MRI following oral glucose tolerance drink. (A) Example of Z-spectra in dynamic scan. (B) the DGE MRI of all subjects with reference to the first time point of the dynamic scan; (C) the dynamic R2* changes of all subjects with reference to the first time point of the dynamic scan. Data (lines) were smoothed with a moving average filter of five points, whereas each data point (circles) were shown as original without any smoothing.

maternal 4.4-4.7 $\mathrm{mM}$ ) (29), the glucose concentration is largely homogenous across the placenta, and therefore GCE might be sensitive to regional pathologies such as infarction and chronic villitis.

The assessment of placental glucose is somewhat confounded by several known factors that may affect the GlucoCEST MRI effect, such as field inhomogeneity and motion $(31,32)$ and factors that are specific to placenta structure, such as volume fractions of fetal and maternal blood. For example, the absolute GCE effect is sensitive to the $\mathrm{B}_{1}$ field. In the human placenta in vivo $(20 \mathrm{~cm}$ in diameter), the $B_{1}$ filed may vary up to $40 \%$, which could translate to $20 \%$ difference in GCE according to previous analysis of CEST effect (25). In addition, the blood sugar level varies throughout the day, whereas the normal range of fasting blood sugar level (3.9-5.5 $\mathrm{mM})$ is close to that of prediabetes $(5.5-7 \mathrm{mM}$ ) and diabetes (more than $7 \mathrm{mM}$ ) (33).
In light of the technical challenges, further development of GCE is needed before it can reliably characterize placental glucose transport. Although the time to peak generally occurs within 2-4 min after IV glucose infusion (21), the time to peak is highly variable following oral glucose drink (34). Despite these challenges, the in vivo DGE showed a consistent trend in all subjects during glucose challenge, which is very encouraging.

It has been shown that apart from the CEST effect, glucose also reduces the water $\mathrm{T} 2$ relaxation due to chemical exchange, which would broaden the water saturation curve $(35,36)$. T2 change of the glucose perfused ex vivo tissue is consistent with the GCE effect, though may be confounded by blood wash out. The in vivo experiments show the $\Delta \mathrm{R} 2$ * increases that are largely similar to the DGE signal, which is confirmative of the glucose enhancement effect.

There are several limitations of the current study, for 
example, the protocol of glucose drink required the subject to sit up and drink the glucose in order to prevent reflux, thus causing inconsistency between baseline measurements and the dynamic measurements. It was also challenging to get a blood sugar level validation, since even a simple serum glucose measure with a finger prick requires the patient to be out of the scan room and sitting down comfortably, which takes about 5-10 min. Thus, the values would not be matched in time. Finally, fetal organs such as fetal livers would be of interest to observe, but accurate assessment of glucose change was challenging due to the fetal motion. In addition, motion caused by maternal free breathing, fetal motion, Braxton-hicks contractions, as well as blood perfusion change induced by increased blood sugar level, needs to be considered in future studies. Quantitative validation of the dynamic GCE signal may also require iv administration for a more rapid bolus, so as to enable correlation between serum glucose and each dynamic scan. Here, we chose to do a simple noninvasive feasibly study as the more invasive study design would need to be justified by a successful feasibility study.

\section{Conclusions}

Our study demonstrated the feasibility of DGE MRI in the human placenta following an oral glucose load at 3.0 Tesla. We found that in vivo DGE showed a consistent trend in all subjects during glucose challenge.

\section{Acknowledgments}

Funding: The study was supported in part by NIH human placenta project U01HD087211 (to Grant) and Shanghai Sailing Program 18YF1410900 (to Luo).

\section{Footnote}

Conflicts of Interest: The authors have no conflicts of interest to declare.

Ethical Statement: The study was approved by the Institutional Review Board at the Boston Children's Hospital and Massachusetts General Hospital.

\section{References}

1. Desoye G, Gauster M, Wadsack C. Placental transport in pregnancy pathologies. Am J Clin Nutr
2011;94:1896S-902S.

2. Close K. Oral Glucose Tolerance Test Uses, Procedure and Results. 2018. Available online: https://www. verywellhealth.com/the-oral-glucose-tolerancetest-1087684

3. Benveniste H, Fowler JS, Rooney WD, Moller DH, Backus WW, Warner DA, Carter P, King P, Scharf B, Alexoff DA, Ma Y, Vaska P, Schlyer D, Volkow ND, study PET, study MRI. Maternal-fetal in vivo imaging: a combined PET and MRI study. J Nucl Med 2003;44:1522-30.

4. Brunelli R, Masselli G, Parasassi T, De Spirito M, Papi M, Perrone G, Pittaluga E, Gualdi G, Pollettini E, Pittalis A, Anceschi MM. Intervillous circulation in intrauterine growth restriction. Correlation to fetal well being. Placenta 2010;31:1051-6.

5. Frias AE, Schabel MC, Roberts VH, Tudorica A, Grigsby PL, Oh KY, Kroenke CD. Using dynamic contrastenhanced MRI to quantitatively characterize maternal vascular organization in the primate placenta. Magn Reson Med 2015;73:1570-8.

6. Zun Z, Zaharchuk G, Andescavage NN, Donofrio MT, Limperopoulos C. Non-Invasive Placental Perfusion Imaging in Pregnancies Complicated by Fetal Heart Disease Using Velocity-Selective Arterial Spin Labeled MRI. Sci Rep 2017;7:16126.

7. Moore RJ, Issa B, Tokarczuk P, Duncan KR, Boulby P, Baker PN, Bowtell RW, Worthington BS, Johnson IR, Gowland PA. In vivo intravoxel incoherent motion measurements in the human placenta using echo-planar imaging at 0.5 T. Magn Reson Med 2000;43:295-302.

8. Moore RJ, Ong SS, Tyler DJ, Duckett R, Baker PN, Dunn WR, Johnson IR, Gowland PA. Spiral artery blood volume in normal pregnancies and those compromised by preeclampsia. NMR Biomed 2008;21:376-80.

9. Moore RJ, Strachan BK, Tyler DJ, Duncan KR, Baker PN, Worthington BS, Johnson IR, Gowland PA. In utero perfusing fraction maps in normal and growth restricted pregnancy measured using IVIM echo-planar MRI. Placenta 2000;21:726-32.

10. Huen I, Morris DM, Wright C, Parker GJ, Sibley CP, Johnstone ED, Naish JH. R1 and R2 * changes in the human placenta in response to maternal oxygen challenge. Magn Reson Med 2013;70:1427-33.

11. Huen I, Morris DM, Wright C, Sibley CP, Naish JH, Johnstone $\mathrm{ED}$. Absence of $\mathrm{PO} 2$ change in fetal brain despite $\mathrm{PO} 2$ increase in placenta in response to maternal oxygen challenge. BJOG 2014;121:1588-94. 
12. Luo J, Abaci Turk E, Bibbo C, Gagoski B, Roberts DJ, Vangel M, Tempany-Afdhal CM, Barnewolt C, Estroff J, Palanisamy A, Barth WH, Zera C, Malpica N, Golland P, Adalsteinsson E, Robinson JN, Grant PE. In Vivo Quantification of Placental Insufficiency by BOLD MRI: A Human Study. Sci Rep 2017;7:3713.

13. Sørensen A, Peters D, Fründ E, Lingman G, Christiansen O, Uldbjerg N. Changes in human placental oxygenation during maternal hyperoxia estimated by blood oxygen level-dependent magnetic resonance imaging (BOLD MRI). Ultrasound Obstet Gynecol 2013;42:310-4.

14. Sørensen A, Sinding M, Peters DA, Petersen A, Frøkjær JB, Christiansen OB, Uldbjerg N. Placental oxygen transport estimated by the hyperoxic placental BOLD MRI response. Physiol Rep 2015. doi: 10.14814/phy2.12582.

15. Denison FC, Semple SI, Stock SJ, Walker J, Marshall I, Norman JE. Novel use of proton magnetic resonance spectroscopy (1HMRS) to non-invasively assess placental metabolism. PLoS One 2012;7:e42926.

16. Kay HH, Gordon JD, Ribeiro AA, Spicer LD. Phosphorus 31 magnetic resonance spectroscopy of human placenta and quantitation with perchloric acid extracts. Am J Obstet Gynecol 1991;164:80-7.

17. Macnaught G, Semple SI, Gray C, Simpson M, Norman JE, Walker J, Denison FC. 1H MRS as a biomarker for placental insufficiency in the growth restricted fetus. 2014. doi:10.1136/archdischild-2014-306576.242.

18. Chan KW, McMahon MT, Kato Y, Liu G, Bulte JW, Bhujwalla ZM, Artemov D, van Zijl PC. Natural D-glucose as a biodegradable MRI contrast agent for detecting cancer. Magn Reson Med 2012;68:1764-73.

19. Walker-Samuel S, Ramasawmy R, Torrealdea F, Rega M, Rajkumar V, Johnson SP, Richardson S, Goncalves M, Parkes HG, Arstad E, Thomas DL, Pedley RB, Lythgoe $\mathrm{MF}$, Golay X. In vivo imaging of glucose uptake and metabolism in tumors. Nat Med 2013;19:1067-72.

20. Xu X, Chan KW, Knutsson L, Artemov D, Xu J, Liu G, Kato Y, Lal B, Laterra J, McMahon MT, van Zijl PC. Dynamic glucose enhanced (DGE) MRI for combined imaging of blood-brain barrier break down and increased blood volume in brain cancer. Magn Reson Med 2015;74:1556-63.

21. Xu X, Yadav NN, Knutsson L, Hua J, Kalyani R, Hall E, Laterra J, Blakeley J, Strowd R, Pomper M, Barker P, Chan K, Liu G, McMahon MT, Stevens RD, van Zijl PC. Dynamic Glucose-Enhanced (DGE) MRI: Translation to Human Scanning and First Results in Glioma Patients. Tomography 2015;1:105-14.
22. Wang J, Weygand J, Hwang KP, Mohamed AS, Ding Y, Fuller CD, Lai SY, Frank SJ, Zhou J. Magnetic Resonance Imaging of Glucose Uptake and Metabolism in Patients with Head and Neck Cancer. Sci Rep 2016;6:30618.

23. Wu D, Xu J, Lei J, McLane M, van Zijl PC, Burd I. Dynamic glucose enhanced MRI of the placenta in a mouse model of intrauterine inflammation. Placenta 2018;69:86-91.

24. van Zijl PCM, Yadav NN. Chemical exchange saturation transfer (CEST): What is in a name and what isn't? Magn Reson Med 2011;65:927-48.

25. Jiang W, Zhou IY, Wen L, Zhou X, Sun PZ. A theoretical analysis of chemical exchange saturation transfer echo planar imaging (CEST-EPI) steady state solution and the CEST sensitivity efficiency-based optimization approach. Contrast Media Mol Imaging 2016;11:415-23.

26. Sun PZ, Lu J, Wu Y, Xiao G, Wu R. Evaluation of the dependence of CEST-EPI measurement on repetition time, RF irradiation duty cycle and imaging flip angle for enhanced pH sensitivity. Phys Med Biol 2013;58:N229-40.

27. Kim M, Gillen J, Landman BA, Zhou J, van Zijl PC. Water saturation shift referencing (WASSR) for chemical exchange saturation transfer (CEST) experiments. Magn Reson Med 2009;61:1441-50.

28. Egbor M, Ansari T, Morris N, Green CJ, Sibbons PD. Morphometric placental villous and vascular abnormalities in early- and late-onset pre-eclampsia with and without fetal growth restriction. BJOG 2006;113:580-9.

29. Holme AM, Roland MC, Lorentzen B, Michelsen TM, Henriksen T. Placental glucose transfer: a human in vivo study. PLoS One 2015;10:e0117084.

30. Luo J, Ji Y, Abaci Turk E, Zhou IY, Roberts DJ, Grant PE, Sun PZ. Feasibility of glucose CEST in the human placenta. Proc Intl Soc Mag Reson Med 2017. Available online: https://cds.ismrm.org/ protected/17MPresentations/abstracts/0104.html

31. Simegn GL, Van der Kouwe AJW, Robertson FC, Meintjes EM, Alhamud A. Real-time simultaneous shim and motion measurement and correction in glycoCEST MRI using double volumetric navigators (DvNavs). Magn Reson Med 2019;81:2600-13.

32. Zaiss M, Herz K, Deshmane A, Kim M, Golay X, Lindig T, Bender B, Ernemann U, Scheffler K. Possible artifacts in dynamic CEST MRI due to motion and field alterations. J Magn Reson 2019;298:16-22.

33. Yasinska IM, Sumbayev VV. S-nitrosation of Cys-800 of HIF-1alpha protein activates its interaction with p300 and stimulates its transcriptional activity. FEBS Lett 
2003;549:105-9.

34. National Center for Health Statistics. The One-hour oral glucose tolerance test. DHEW Publication, 1973.

35. Gore JC, Brown MS, Mizumoto CT, Armitage IM. Influence of glycogen on water proton relaxation times.

Cite this article as: Luo J, Abaci Turk E, Gagoski B, Copeland N, Zhou IY, Young V, Bibbo C, Robinson JN, Zera C, Barth WH Jr, Roberts DJ, Sun PZ, Grant PE. Preliminary evaluation of dynamic glucose enhanced MRI of the human placenta during glucose tolerance test. Quant Imaging Med Surg 2019;9(10):1619-1627. doi: 10.21037/ qims.2019.09.16
Magn Reson Med 1986;3:463-6.

36. Yadav NN, Xu J, Bar-Shir A, Qin Q, Chan KW, Grgac K, Li W, McMahon MT, van Zijl PC. Natural D-glucose as a biodegradable MRI relaxation agent. Magn Reson Med 2014;72:823-8. 